BULL. AUSTRAL. MATH. SOC.

$30 A 95,39 A 05$

VOL. $10(1974), 281-291$.

\title{
A new definition of discrete analytic functions
}

\author{
C.J. Harman
}

\begin{abstract}
The concept of a tetradiffric function is introduced. This new scheme for defining discrete analytic functions is shown to retain the algebraic simplicity of monodiffric functions, while introducing to the theory a symmetry similar to the Schwarz Reflection Principle.
\end{abstract}

\section{Introduction and definitions}

Discrete analytic functions of the first kind (or monodiffric functions) are defined on the set of gaussian integers and satisfy the forward-difference equation

$$
f(z+1)-f(z)=\frac{f(z+i)-f(z)}{i},
$$

(see for example Isaacs $[7,8]$ and Berzsenyi $[1,2]$ ). In [6], the monodiffric function $z^{(\alpha)}$ (the discrete analogue of $z^{\alpha}$ ) was found. This function highlighted certain shortcomings in the monodiffric scheme. Monodiffric functions lack symmetry: for example $(-z)^{(\alpha)} \neq(-1)^{\alpha}{ }^{(\alpha)}$, and in the theory there is no analogue of the Schwarz Reflection Principle.

In this paper an alternative definition of discrete analytic functions is examined. The resulting functions demonstrate a symmetry similar to discrete functions of the second kind which were defined by Ferrand [4] and further developed by Duffin [3] and others. Unlike second kind functions however, it is seen that the simple algebraic form of monodiffric functions

Received 27 November 1973. 
is retained. The function $z^{(\alpha)}$ is expressed in terms of divergent series when $\alpha$ is not an integer and as a polynomial when $\alpha$ is a non-negative integer. Finally an analogue of the Schwarz Reflection Principle is obtained.

The domain of definition to be considered is the set $G$ of gaussian integers. Hence,

$$
G=\{z ; z=(x, y)=x+i y \text {, where } x \text { and } y \text { are integers }\} .
$$

Subsets of $G$ in the four quadrants of the complex plane are defined by,

$$
\begin{aligned}
& G_{1}=\{z ; z \in G, x>0, y>0\}, G_{2}=\{z ; z \in G, x<0, y>0\}, \\
& G_{3}=\{z ; z \in G, x<0, y<0\}, G_{4}=\{z ; z \in G, x>0, y<0\},
\end{aligned}
$$

and on the axes,

$$
\begin{aligned}
& X^{+}=\{z ; z \in G, x \geq 0, y=0\}, X^{-}=\{z ; z \in G, x \leq 0, y=0\}, \\
& Y^{+}=\{z ; z \in G, x=0, y \geq 0\}, Y^{-}=\{z ; z \in G, x=0, y \leq 0\} .
\end{aligned}
$$

Forward and backward difference operators are defined by,

$$
\begin{aligned}
& \Delta_{1} f(z)=f(z)-f(z-1), \\
& \Delta_{2} f(z)=\frac{f(z)-f(z-i)}{i}, \\
& \Delta_{3} f(z)=f(z+1)-f(z), \\
& \Delta_{4} f(z)=\frac{f(z+i)-f(z)}{i} .
\end{aligned}
$$

\section{Tetradiffric functions}

A new type of discrete analytic function, based on the concept of a monodiffric function, is now defined. The definition involves a consideration of a separate monodiffric scheme in each of the four quadrants $G_{1}, G_{2}, G_{3}$, and $G_{4}$.

A function $f$ is said to be tetradiffric at the point $z \in G_{k}$ $(k=1,2,3$, or 4$)$, if 


$$
\Delta_{k} f(z)=\Delta_{k+1} f(z)
$$

(For convenience of notation it has been assumed that in the case when $k=4$, the operator $\Delta_{5}$ means $\Delta_{1}$.)

The importance of this method of definition is illustrated by the following theorem:- a tetradiffric function can be represented in any of the four quadrants of the complex plane by a linear combination of values from both the $X$ and $Y$ axes.

THEOREM 2.1. The unique tetradiffric function $f$, with values prescribed on the axes (on $X^{+} \cup X^{-} \cup Y^{+} \cup Y^{-}$) is given by the following:(i) if $z=(x, y) \in G_{1}$,

$$
\begin{aligned}
f(z)=(1-i)^{-(x+y)}\left\{\sum_{j=0}^{x}\left(\begin{array}{c}
x+y \\
j
\end{array}\right)(-i)^{j}\left(1-i \Delta_{1}\right)^{x-j} f(x-j, 0)\right. & \\
& \left.+\sum_{j=x+1}^{x+y}\left(\begin{array}{c}
x+y \\
j
\end{array}\right)(-i)^{j}\left(1-\Delta_{2}\right)^{j-x} f(0, j-x)\right\} ;
\end{aligned}
$$

(ii) if $z=(x, y) \in G_{2}$,

$$
\begin{aligned}
f(z)=(1+i)^{x-y}\left\{\sum_{j=0}^{-x}\left(\begin{array}{c}
y-x \\
j
\end{array}\right) i^{j}\left(1-i \Delta_{3}\right)^{-x-j} f(x+j, 0)\right. & \\
& \left.+\sum_{j=1-x}^{y-x}\left(\begin{array}{c}
y-x \\
j
\end{array}\right) i^{j}\left(1+\Delta_{2}\right)^{x+j} f(0, x+j)\right\} ;
\end{aligned}
$$

(iii) if $z=(x, y) \in G_{3}$,

$f(z)=(1-i)^{x+y}\left\{\sum_{j=0}^{-x}\left(\begin{array}{c}-x-y \\ j\end{array}\right)(-i)^{j}\left(1+i \Delta_{3}\right)^{-x-j} f(x+j, 0)\right.$

$$
\left.+\sum_{j=1-x}^{-x-y}\left(\begin{array}{c}
-x-y \\
j
\end{array}\right)(-i)^{j}\left(1+\Delta_{4}\right)^{x+j} f(0,-x-j)\right\}
$$

(iv) if $z=(x, y) \in G_{4}$, 


$$
\begin{aligned}
f(z)=(1+i)^{y-x}\left\{\sum_{j=0}^{x}\left(\begin{array}{c}
x-y \\
j
\end{array}\right) i^{j}\left(1+i \Delta_{1}\right)^{x-j} f(x-j, 0)\right. & \\
& \left.+\sum_{j=x+1}^{x-y}\left(\begin{array}{c}
x-y \\
j
\end{array}\right) i^{j}\left(1-\Delta_{4}\right)^{j-x} f(0, x-j)\right\} .
\end{aligned}
$$

The binomial operators in the above are defined in the usual way; for example

$$
\left(1-i \Delta_{1}\right)^{x-j}=\sum_{j=0}^{x-j}\left(\begin{array}{c}
x-j \\
k
\end{array}\right)(-i)^{k} \Delta_{1}^{k} ;\left(1-i \Delta_{1}\right)^{0}=I,
$$

where $I$ is the identity operator.

The proof of (i) above follows from [6, Theorem 2.3] and (ii), (iii), (iv) are proved in a similar way.

Hence a tetradiffric function $f(z)$ can be expressed in terms of a combination of specified values on the two half-axes which bound the quadrant containing the point $z$.

For example consider two simple cases:- from the above theorem it follows that for $z=(1,1) \in G_{1}$,

$$
f(1,1)=(1-i)^{-1}[f(1,0)-i f(0,1)] \text {, }
$$

and for $z=(2,-1) \in G_{4}$,

$$
f(2,-1)=(1+i)^{-3}[2 i f(2,0)+(i-1) f(1,0)-(1+i) f(0,-1)] \text {. }
$$

\section{The tetradiffric function $z^{(\alpha)}$}

The monodiffric function $z^{(\alpha)}$ ( $\alpha$ not a negative integer), given in [6], is now extended to tetradiffric functions. The resulting function highlights some important advantages of the tetradiffric scheme.

For points on the $x$-axis, the function $x^{(\alpha)}$ is to be defined by

$$
x^{(\alpha)}= \begin{cases}\frac{\Gamma(x+\alpha)}{\Gamma(x)} ; & x \in X^{+}, \\ \frac{(-1)^{\alpha} \Gamma(\alpha-x)}{\Gamma(-x)} ; & x \in X^{-},\end{cases}
$$


and on the $Y$-axis

$$
(i y)^{(\alpha)}=i_{y}^{(\alpha)} ; \quad i y \in Y^{+} \cup Y^{-}
$$

where $y^{(\alpha)}$ is given by (3.1).

Note that $x^{(\alpha)}$ satisfies $\Delta_{1} x^{(\alpha)}=\alpha x^{(\alpha-1)}$ for $x \in X^{+}$, and $\Delta_{3} x^{(\alpha)}=\alpha x^{(\alpha-1)}$ for $x \in X^{-}$. Also it can be shown that $x^{(\alpha)}$ is a very good asymptotic approximation to $x^{\alpha}$ on both $x^{+}$and $X^{-}$.

The tetradiffric analogue $z^{(\alpha)}$ of the classical function $z^{\alpha}$ is required to satisfy

$$
\begin{aligned}
& \text { (i) } \Delta z^{(\alpha)}=z^{(\alpha-1)} \text {, } \\
& \text { (ii) } 0^{(\alpha)}=0, \alpha>0 \text {, and } \\
& \text { (iii) } z^{(0)}=1 \text {, }
\end{aligned}
$$

where $\Delta=\Delta_{k}$ or $\Delta_{k+1}$ for $z \in G_{k} ; k=1,2,3,4$.

The case when $\alpha=n$, a non-negative integer, is quite simple. It can be shown that the function $z^{(n)}$ given by,

$$
z^{(n)}=\sum_{j=0}^{n}\left(\begin{array}{l}
n \\
j
\end{array}\right) x^{(n-j)} i^{j} y^{(j)} ; z^{(0)}=1,
$$

is the tetradiffric function satisfying (3.3) and having the values $x^{(n)}$ and $(i y)^{(n)}$ on the axes.

When $\alpha$ is a negative integer, the function $x^{(\alpha)}$ has singularities at certain points on $X^{+} \cup X^{-}$. It will now be assumed that $\alpha$ is not an integer, but is otherwise an arbitrary constant.

By specifying $x^{(\alpha)}$ and $(i y)^{(\alpha)}$ on the axes, Theorem 2.1 provides the tetradiffric function $z^{(\alpha)}$ at any point in $G$, and as in [6, Theorem 3.1] it can easily be shown that $z^{(\alpha)}$ satisfies conditions (i) and (ii) of (3.3). However the resulting function $z^{(\alpha)}$ has a rather complicated 
form, and an alternative expression is now derived which has a remarkable analogy with the binomial expansion of the function $z^{\alpha}=(x+i y)^{\alpha}$.

THEOREM 3.1. If $z=(x, y) \in G$ and $x^{(\alpha)}, y^{(\alpha)}$ are defined by (3.1) then the tetradiffric function $z^{(\alpha)}$ is given by

$$
z^{(\alpha)}=\sum_{j=0}^{\infty}\left(\begin{array}{l}
\alpha \\
j
\end{array}\right) x^{(\alpha-j)} i^{j} y^{(j)}+\sum_{j=0}^{\infty}\left(\begin{array}{l}
\alpha \\
j
\end{array}\right) x^{(j)} i^{\alpha-j} y^{(\alpha-j)},
$$

where the two divergent series are sumable $(E, q)$ in the Euler sense for $q>0$.

Proof. Define a function $z^{(\alpha)}$ by

$$
z^{(\alpha)}=\sum_{j=0}^{\infty}\left(\begin{array}{l}
\alpha \\
j
\end{array}\right) x^{(\alpha-j)} i^{j} y^{(j)}+\sum_{j=0}^{\infty}\left(\begin{array}{l}
\alpha \\
j
\end{array}\right) x^{(j)} i^{\alpha-j} y^{(\alpha-j)},
$$

and let $z=(x, y) \in G_{1}$. For convenience consider the first of the above two sums and denote it by

$$
S_{\alpha}(z)=\sum_{j=0}^{\infty} a_{j} \text {, where } a_{j}=\left(\begin{array}{l}
\alpha \\
j
\end{array}\right) x^{(\alpha-j)} i^{j} y^{(j)} .
$$

Now it can easily be verified that for $z \in G_{1}$, the series $S_{\alpha}(z)$ diverges. For $S_{\alpha}$ to be summable $(E, q)$ it must be shown that

(a) $\sum_{j=0}^{\infty} a_{j} \rho^{j+1}$ converges for small $\rho$, and

(b) the series $s_{\alpha}(z)$ defined by

$$
s_{\alpha}(z)=\sum_{n=0}^{\infty}(1+q)^{-n-1} \sum_{j=0}^{n}\left(\begin{array}{l}
n \\
j
\end{array}\right) \alpha_{j} q^{n-j}
$$

converges (see Hardy [5]).

If these conditions hold, the series $S_{\alpha}$ is said to be sumable $(E, q)$ to the sum $s_{\alpha}$. That condition (a) holds in this case is readily checked. The following lemma shows that (b) is true.

LEMMA 3.1. For $z=(x, y) \in G_{1}$, the series defined by 


$$
s_{\alpha}(z)=\sum_{n=0}^{\infty}(1+q)^{-n-1} \sum_{j=0}^{n}\left(\begin{array}{l}
n \\
j
\end{array}\right)\left(\begin{array}{l}
\alpha \\
j
\end{array}\right) x^{(\alpha-j)} i^{j} y^{(j)} q^{n-j}
$$

converges absolutely for $q>0$.

Proof. For $z \in G_{I}$ it follows from the definitions of $x^{(\alpha)}$ and $y^{(\alpha)}$ that

$$
\left(\begin{array}{l}
\alpha \\
j
\end{array}\right) x^{(\alpha-j)} y^{(j)}=\frac{(\alpha-j+1)(\alpha-j+2) \ldots(\alpha-j+x-1)(j+1)(j+2) \ldots(j+y-1)}{\Gamma(x) \Gamma(y)} .
$$

This is a polynomial in $j$ of degree $(x+y-2)$, and can be writ: en as

$$
\left(\begin{array}{l}
\alpha \\
j
\end{array}\right) x^{(\alpha-j)} y^{(j)}=\sum_{k=0}^{x+y-2} b_{k^{j}}
$$

where the coefficients $b_{k}$ are determined by $x, y$ and $\alpha$. Hence $s_{\alpha}(z)$ becomes;

$$
s_{\alpha}(z)=\sum_{n=0}^{\infty}(1+q)^{-n-1} \sum_{k=0}^{x+y-2} b_{k} \sum_{j=0}^{n}\left(\begin{array}{l}
n \\
j
\end{array}\right) q^{n-j} i^{j} j^{k} .
$$

Now it can readily be shown by induction on $k$ that for fixed $n$,

$$
\sum_{j=0}^{n}\left(\begin{array}{l}
n \\
j
\end{array}\right) q^{n-j} i_{j}^{j k}= \begin{cases}(q+i)^{n} & ; k=0, \\
\sum_{r=1}^{k} \frac{s_{r}^{(k)} n ! i^{r}(q+i)^{n-r}}{(n-r) !} ; k \geq 1,\end{cases}
$$

where $S_{r}^{(k)}$ are Stirling numbers of the second kind.

Hence, assuming for the moment that summation can be interchanged,

$$
s_{\alpha}(z)=b_{0} \sum_{n=0}^{\infty} \frac{(q+i)^{n}}{(q+1)^{n+1}}+\sum_{k=1}^{x+y-2} b_{k} \sum_{r=1}^{k} \frac{s_{r}^{(k)} i^{r}}{(q+1)^{r+1}} \sum_{n=0}^{\infty} \frac{n !}{(n-r) !}\left(\frac{q+i}{q+1}\right)^{n-r} \text {, }
$$

and since $\left|\frac{q+i}{q+1}\right|<1$ when $q>0$, it follows that the above series are absolutely convergent, which justifies the interchange of summation and proves the lexme.

Returning to the proof of the theorem; it has been shown that $s_{\alpha}(z)$ 
converges and hence by $(a)$ and (b) above, $S_{\alpha}(z)$ is summable $(E, q)$ for $q>0$ to the sum $s_{\alpha}(z)$.

Similarly the second series in (3.5), $\sum_{j=0}^{\infty}\left(\begin{array}{l}\alpha \\ j\end{array}\right) x^{(j)} i^{\alpha-j} y^{(\alpha-j)}$ is. summable $(E, q), q>0$.

Now by $(1.1)$;

$$
\begin{aligned}
\Delta_{1} z^{(\alpha)}= & z^{(\alpha)}-(z-1)^{(\alpha)} \\
= & \sum_{j=0}^{\infty}\left(\begin{array}{l}
\alpha \\
j
\end{array}\right) x^{(\alpha-j)} i^{j} y^{(j)}-\sum_{j=0}^{\infty}\left(\begin{array}{l}
\alpha \\
j
\end{array}\right)(x-1)^{(\alpha-j)} i_{y}{ }^{(j)} \\
& +\sum_{j=0}^{\infty}\left(\begin{array}{l}
\alpha \\
j
\end{array}\right) x^{(j)} i^{\alpha-j} y^{(\alpha-j)}-\sum_{j=0}^{\infty}\left(\begin{array}{l}
\alpha \\
j
\end{array}\right)(x-1)^{(j)} i^{\alpha-j} y^{(\alpha-j)},
\end{aligned}
$$

and by Hardy [5, p. 180 , Properties $\alpha, \beta]$ it follows that

$$
\begin{aligned}
\Delta_{1} z^{(\alpha)} & =\alpha \sum_{j=0}^{\infty}\left(\begin{array}{c}
\alpha-1 \\
j
\end{array}\right) i_{y}^{j(j)} x^{(\alpha-1-j)}+\alpha \sum_{j=0}^{\infty}\left(\begin{array}{c}
\alpha-1 \\
j
\end{array}\right) i^{\alpha-1-j} y^{(\alpha-1-j)} x^{(j)} \\
& =\alpha z^{(\alpha-1)}
\end{aligned}
$$

Similarly $\Delta_{2} z^{(\alpha)}=\alpha z^{(\alpha-1)}$ and so the function $z^{(\alpha)}$ is tetradiffric for $z \in G_{1}$. It evidently satisfies $0^{(\alpha)}=0$.

On the axes, $z^{(\alpha)}=x^{(\alpha)}$ when $y=0$, and $z^{(\alpha)}=i^{\alpha} y^{(\alpha)}$ when $x=0$. Hence by Theorem 2.1, $z^{(\alpha)}$ is the unique tetradiffric function in $G_{1}$ with prescribed values $x^{(\alpha)}$ on $x^{+}$and $(i y)^{(\alpha)}$ on $y^{+}$.

In a similar manner it can be shown that (3.5) represents the tetradiffric analogue of $z^{\alpha}$ in the other three quadrants $G_{2}, G_{3}$ and $G_{4}$. This completes the proof of Theorem 3.1.

As an example of the method of Euler summability in the above theorem, consider the simple case $z=1+i$. From (3.5), 


$$
\begin{aligned}
& z^{(\alpha)}=(1,1)^{(\alpha)}=\sum_{j=1}^{\infty}\left(\begin{array}{l}
\alpha \\
j
\end{array}\right) 1^{(\alpha-j)} i_{1}{ }^{(j)}+\sum_{j=0}^{\infty}\left(\begin{array}{l}
\alpha \\
j
\end{array}\right){ }_{1}^{(j)} i^{\alpha-j}{ }_{1}(\alpha-j) \\
& =I^{(\alpha)} \sum_{j=0}^{\infty} i^{j}+1^{(\alpha)} \sum_{j=0}^{\infty} i^{\alpha-j} \text {. }
\end{aligned}
$$

Defining $S$ by

$$
S \equiv \sum_{j=0}^{\infty} i^{j}=1+i-1-i+1+i-1-i+\ldots,
$$

then by Hardy $[5, p .180$, Properties $\gamma, \delta]$ it follows that

$$
\begin{aligned}
S & =1+i(1+i-1-i+1+\ldots) \\
& =1+i S
\end{aligned}
$$

and so $S=(1-i)^{-1}$. Similarly

$$
\sum_{j=0}^{\infty} i^{\alpha-j}=-i^{\alpha+1}(1-i)^{-1}
$$

and hence $(1,1)^{\alpha}=1^{(\alpha)}\left(1-i^{\alpha+1}\right)(1-i)^{-1}$, which checks with (2.2) on substituting $f(1,0)=1^{(\alpha)}, f(0,1)=i_{1}^{\alpha}(\alpha)$.

\section{Properties}

When $\alpha$ is not an integer, the tetradiffric function $z^{(\alpha)}$ given by (3.5) is evidently multi-valued. This demonstrates a good analogy with the classical function $z^{\alpha}$.

Also by making use of backward differences on the positive half axes and forward differences on the negative half axes, the function $z^{(\alpha)}$ can be shown to be a very good approximation to $z^{\alpha}$ on $X^{+} \cup X^{-} \cup Y^{+} \cup Y^{-}$, even for small integer values of $x$ and $y$.

The Schwarz Reflection Principle has an analogy for the tetradiffric function $z^{(\alpha)}$ as is indicated in the following theorem.

THEOREM 4.1. When $\alpha$ is real, the tetradiffric function $z^{(\alpha)}$ is real for $z \in X^{+}$and satisfies the symmetry condition $z^{(\alpha)}=\overline{(\bar{z})^{(\alpha)}}$ for 
$z \in G_{1} \cup G_{4}$.

Proof. Let $z=(x, y) \in G_{1}$. By $(3.4)$,

$$
(\bar{z})^{(\alpha)}=(x,-y)^{(\alpha)}=\sum_{j=0}^{\infty}\left(\begin{array}{l}
\alpha \\
j
\end{array}\right) x^{(\alpha-j)} i^{j}(-y)^{(j)}+\sum_{j=0}^{\infty}\left(\begin{array}{l}
\alpha \\
j
\end{array}\right) x^{(j)} i^{\alpha-j}(-y)^{(\alpha-j)} .
$$

Since iy $\in Y^{+},-i y \in Y^{-}$it follows from (3.1), (3.2) that

$$
(-y)^{(\alpha)}=(-1)^{\alpha} \frac{\Gamma(\alpha+y)}{\Gamma(y)}=(-1)^{\alpha} y^{(\alpha)} \text {. }
$$

Hence

$$
(\bar{z})^{(\alpha)}=\sum_{j=0}^{\infty}\left(\begin{array}{l}
\alpha \\
j
\end{array}\right) x^{(\alpha-j)}(-i)^{j} y^{(j)}+\sum_{j=0}^{\infty}\left(\begin{array}{l}
\alpha \\
j
\end{array}\right) x^{(j)}(-i)^{\alpha-j} y^{(\alpha-j)},
$$

and since $x^{(\alpha)}, y^{(\alpha)}$ are real for real $\alpha$ and $x \geq 0, y \geq 0$, it follows that

$$
\overline{(\bar{z})^{(\alpha)}}=z^{(\alpha)} \text {. }
$$

If $z \in G_{4}$ the above argument can be reversed, proving the theorem.

Another important property of $z^{(\alpha)}$ which demonstrates once again the symmetry of tetradiffric functions is given by the following.

THEOREM 4.2. FOr $z \in G$,

$$
(-z)^{(\alpha)}=(-1)^{\alpha} z^{(\alpha)}
$$

The proof follows immediately from (3.1), (3.2), and (3.5).

Theorem 4.1 can be generalized to a wider class of tetradiffric functions as follows.

THEOREM 4.3. If $f$ is a tetradiffric function which is real on the $X$-axis and such that $\overline{f(\bar{z})}=f(z)$ for $z \in Y^{+} \cup Y^{-}$, then for all $z \in G$,

$$
\overline{f(\bar{z})}=f(z) \text {. }
$$

The proof follows readily from Theorem 2.1 and so is omitted.

When $\alpha=n$ a non-negative integer, Theorems 4.1 and 4.2 also apply to the function $z^{(n)}$ given by $(3.4)$. 
For convenience it has been assumed throughout this paper that the functions concerned are tetradiffric on all of $G$. This restriction can of course be weakened to a consideration of functions tetradiffic on smaller domains.

\section{References}

[1] George Berzsenyi, "Line integrals for monodiffric functions", $J$. Math. Anal. App 2. 30 (1970), 99-112.

[2] George Berzsenyi, "Convolution products of monodiffric functions", $J$. Math. Anal. Appl. 37 (1972), 271-287.

[3] R.J. Duffin, "Basic properties of discrete analytic functions", Duke Math. J. 23 (1956), 335-363.

[4] Jacqueline Ferrand, "Fonctions préharmoniques et fonctions préholomorphes", Bulz. Sci. Math. (2) 68 (1944), 152-180.

[5] G.H. Hardy, Divergent series (Clarendon Press, Oxford, 1949).

[6] C.J. Harman, "A note on a discrete analytic function", Buzl. Austral. Math. Soc. 10 (1974), 123-134.

[1] Rufus Philip Isaacs, "A finite difference function theory", Univ. Nac. Tucumán Rev. Ser. A 2 (1941), 177-201.

[8] Rufus Isaacs, "Monodiffric functions", Construction and applications of conformal maps, 257-266 (Proc. Sympos. 1949, Numerical analysis, National Bureau of Standards, Univ. California, Los Angeles. National Bureau of Standards Applied Mathematics Series, 18. United States Department of Commerce; US Government Printing Office, Washington, DC, 1952).

Department of Supply,

Weapons Research Establishment,

Salisbury,

South Australia. 